\title{
FINITE ELEMENT MODELLING FOR THE OPTIMIZATION OF MICROHEATING IN DISPOSABLE MOLECULAR DIAGNOSTICS
}

\author{
T. PARDY ${ }^{1}$, T. RANG ${ }^{1} \&$ I. TULP ${ }^{2}$ \\ ${ }^{1}$ Thomas Johann Seebeck Department of Electronics, Tallinn University of Technology, Estonia. \\ ${ }^{2}$ SelfDiagnostics Deutschland GmbH, Germany.
}

\begin{abstract}
The number of disposable molecular diagnostics tests in the IVD market has been growing rapidly and is bound to continue to grow in the near future. The internal complexity of these rapid tests increases with the complexity of the diagnostic assay implemented by them. Some assays require precise temperature control $\left( \pm 1^{\circ} \mathrm{C}-5^{\circ} \mathrm{C}\right)$ for an extended time (i.e. $15-60$ minutes) for the reactions involved to run properly. Microheating components in them must meet strict criteria with respect to power consumption, physical size and cost. The proposed finite element model is intended to provide tools for in silico validation of device designs (geometries, structural materials), as well as to help in the interpretation of heat transfer processes inside the thermal system present in a molecular diagnostics device. The proposed model was developed for and validated with polyimide etched foil heating elements actively controlled via a mini-thermostat. The thermostat was designed for battery-based operation and implemented with open-source hardware (Arduino-compatible). Plastic test structures were created that emulated disposable Lab-on-a-Chip devices with microfluidic channels to hold liquid volumes on the scale of $0.1 \mathrm{~mL}$. The experimental setup was demonstrated to maintain target temperatures over at least 30 minutes with at least $\pm 1^{\circ} \mathrm{C}$ around the set point operated from batteries. Physical parameters of the resistive heating element used were fed into the finite element model and simulation results compared to the performance of the aforementioned experimental setup.
\end{abstract}

Keywords: computer aided design, finite element modelling, lab-on-a-chip, microfluidics, microcontrollers, resistive heating.

\section{INTRODUCTION}

In molecular diagnostics, temperature control is required for a wide variety of assays, particularly immunoassays and nucleic acid amplification assays. These assays require complex liquid handling, usually done via microfluidics. For a portable, disposable device, it is necessary to have a heating solution with low power dissipation, minimal space requirements and low cost per device. Electrical heaters easily satisfy these criteria. To integrate electrical heating into a portable, handheld device, device geometry must be optimized to minimize heat loss and maximize power efficiency. In silico validation of CAD models using finite element models is an attractive option for optimization, enabling iterative design while saving time and prototyping costs.

Existing works in the literature primarily deal with the finite element modelling of heat transfer and microheating in microfluidic Lab-on-a-Chip systems, primarily with a focus on micromachined thin film resistive heaters and microfluidic chips, as well as with a focus on PCR (polymerase chain reaction), a nucleic amplification method that requires thermal cycling, a challenging task in a portable chip format. Moschou [1] used finite element models to simulate the functionality of their PCR chip, which relied on a thin film heater for temperature control. Wang [2] proposed a Reduced Order Model for simulating microfluidic PCR chips. Models of thermoelectric heating (i.e. Peltier) elements are also discussed in the 
literature but are outside the scope of our work as their unit cost is typically higher than that of resistive heaters, making them less attractive for disposable applications.

However, works where model disposable Point-of-Care devices that rely on isothermal nucleic acid amplification methods were not possible to find. Models presented in the literature typically focus on certain aspects of a device, such as a microreactor or a microfluidic chip, rather than its entirety, disregarding additional device components, such as the housing that plays a significant part in heat transfer phenomena in the size regime of such devices. For iterative design and in silico validation, a complete device model is necessary, and currently available hardware and software make such models possible.

Earlier we demonstrated finite element models for passively regulated (self-regulating) and non-regulated resistive heating for temperature control in disposable Point-of-Care rapid tests using commercially available resistive heating elements [3]. In this paper, we propose a finite element model for active temperature control of disposable molecular diagnostics devices, as well as demonstrate a mini-thermostat application based on open-source (Arduinocompatible) hardware for various isothermal nucleic acid amplification assays. The proposed finite element model was tested for an experimental setup that emulated microfluidics-based molecular diagnostics devices, using a commercially available heating element. The experimental setup was demonstrated to maintain target temperatures over 30 minutes with at least $\pm 1^{\circ} \mathrm{C}$ precision around the set point. Battery-based operation was also evaluated.

\section{THEORETICAL BACKGROUND}

\subsection{Temperature control algorithm}

Active control in the proposed system relies on the proportional control algorithm, expressed in the following way [4]:

$$
V_{\text {in }}(t)=K_{p} \cdot e(t)
$$

where $V_{\text {in }}[V]$ is the potential drop on the heating element, $K_{p}[1 / K]$ the proportional controller gain, and $e(t)=T_{\text {set }}-T_{\text {current }}$ the process error at time instant $t[s] . T_{\text {set }}[K]$ is the temperature set point of the control algorithm, $T_{\text {current }}[K]$ the measured temperature at time instant $t$. Proportional control is sufficient to achieve the targeted $\pm 1^{\circ} \mathrm{C}$ precision while being simpler to implement in a finite element model than a full PID controller.

\subsection{Finite element modelling}

The basis for modelling resistive heating is provided by Joule's first law, which states that electric currents passing through a conductor generate heat, which is proportional to the resistance of the conductor $R[\Omega]$ and the current $I[\mathrm{~A}]$ passing through it:

$$
Q \propto I^{2} \cdot R
$$

$Q[\mathrm{~W}]$ is the energy (heat) released, and if direct current is applied, can be expressed via the formula of power: $P=V \cdot I=I^{2} \cdot R$. The initial assumption is made that liquid in the microchannel is either slow enough to be considered stationary, or is kept stationary for the duration of heating.

Therefore, the model consists of two equations: heat generation is expressed by the formula of power eqn (3), and then heat is propagated through the geometry of the model by 
the heat transfer equation, eqn (6). Space-dependence in the model is defined by a Cartesian coordinate system with 3 axes $x[\mathrm{~m}], y[\mathrm{~m}], z[\mathrm{~m}]$, whereas time-dependence is described by time $(t)$ in seconds. Scaling is permitted. Heat generation is expressed via the aforementioned formula of power, written in a differential form for the volume of the heater [5]:

$$
\frac{d P}{d V}=J \cdot E=J \cdot \frac{J}{\sigma}=J^{2} \cdot \rho
$$

where $P[\mathrm{~W}]$ is the power, $\mathrm{V}\left[\mathrm{m}^{3}\right]$ the volume of the heating element, $\mathrm{J}\left[\mathrm{A} / \mathrm{m}^{2}\right]$ the current density, $\mathrm{E}[\mathrm{V} / \mathrm{m}]$ the electric field. $\sigma[\mathrm{S} / \mathrm{m}]$ is the conductivity and $\rho[\Omega \cdot \mathrm{m}]$ is the resistivity of the material of the heater. Current densities and the electric field used for calculating heat generation are in turn calculated from the following set of equations [6]:

$$
\begin{gathered}
\nabla J=Q_{j}, \\
J=\left(\sigma+\varepsilon_{0} \varepsilon_{r} \frac{\partial}{\partial t}\right) E+J_{e}, \\
E=-\nabla V .
\end{gathered}
$$

where $\mathrm{J}\left[\mathrm{A} / \mathrm{m}^{2}\right]$ is the current density, $Q_{j}\left[\mathrm{~A} / \mathrm{m}^{3}\right]$ are current sources (sinks), $E$ the electric field, $J_{e}\left[\mathrm{~A} / \mathrm{m}^{2}\right]$ an external current density (if there is one), $V[\mathrm{~V}]$ the potential drop in every direction, $\sigma[\mathrm{S} / \mathrm{m}]$ the conductivity, $\varepsilon_{0}$ the relative permittivity of vacuum and $\varepsilon_{r}$ the relative permittivity of the material at the point of the geometry for which the equations are solved. The controller algorithm (Section 2.1) is coupled with the electric field equation eqn (4) as a boundary condition $V=V_{\text {in }}$ at the input terminal. Materials in the simulated geometry are characterized electrically through their conductivity and relative permittivity. The above set of equations eqn (4) relies on the continuity equation, or charge conservation principle [6]:

$$
\nabla J=-\frac{\partial p}{\partial t}
$$

The continuity equation states that charge density at a point changes only if current flows into or out of the point.

The heat transfer equation is used in the following form [7]:

$$
\rho C_{p} \frac{\partial T}{\partial t}-\nabla \cdot(-\mathrm{k} \nabla \mathrm{T})=\mathrm{Q}
$$

where $\rho\left[\mathrm{kg} / \mathrm{m}^{3}\right]$ is the fluid density, $C_{p}[\mathrm{~J} / \mathrm{kg} \mathrm{K}]$ the specific heat capacity at constant pressure, $T[\mathrm{~K}]$ the absolute temperature in the system, $k[\mathrm{~W} /(\mathrm{mK})]$ the thermal conductivity and $\mathrm{Q}\left[\mathrm{W} / \mathrm{m}^{3}\right]$ is the heat source (sink), which in this case is calculated as the power of the heating element as a whole. With respect to heat transfer, materials of the simulated geometry are simulated through the aforementioned quantities $\left(\rho, C_{p}, k\right)$, solved for each spatial point in the geometry.

In case the temperature dependence of resistivity is taken into account, the well-known formula for calculating temperature dependent resistivity defines changes [8]:

$$
\rho(T)=\rho_{0} \cdot\left[1+\alpha \cdot\left(T-T_{0}\right)\right]
$$


where $\rho_{0}[\Omega \cdot m]$ is the resistivity at ambient (reference) temperature $T_{0}[K] . T[K]$ is the current temperature of the heating element, calculated from the heat transfer equation, and $\alpha\left[\frac{1}{K}\right]$ is the temperature coefficient of resistivity. Resistivity is derived from resistance in an experimental setup using the following formula [5]:

$$
R=\rho \frac{l}{A}
$$

Where $l(\mathrm{~m})$ is the length (thickness) of the resistive element, and $A\left(\mathrm{~m}^{2}\right)$ its cross-sectional area. This formula stands for resistors with uniform cross-sectional area, which is true for all of the devices used in the experimental setups discussed in this paper.

\section{EXPERIMENTAL SETUP FOR MODEL VALIDATION}

\subsection{Mini-thermostat}

The mini-thermostat prototype (Fig. 1a) was created on open-source hardware to support isothermal nucleic acid amplification reactions in disposable Point-of-Care/Lab-on-a-Chip devices, preferably operated from a portable power source. Two main goals in mind were simplicity (ease of use) and scalability (downscaling to fit under chips of 5-6 $\mathrm{cm}^{2}$ area and less powerful microcontroller chips).

The platform was first implemented on an Arduino Uno microcontroller (ATmega328P) board with an added custom circuit to handle temperature input from a 2-wire $10 \mathrm{k} \Omega$ NTC (negative temperature coefficient) thermistor and control current output via a MOSFET (metal-oxide semiconductor field-effect transistor). The controller was equipped with a piezo buzzer for auditory feedback to the user about its operation. Additionally, a graphical user interface was developed (Fig. 1b) based on the Microsoft .NET framework for easily updating control parameters and monitoring operation through the serial interface of the Arduino platform using a USB connection on the computer.

\subsection{Setups \#1 and \#2}

In both experimental setups, a commercially available flexible etched foil heating element was used. These resistive heating elements are typically composed of a thin metal film etched
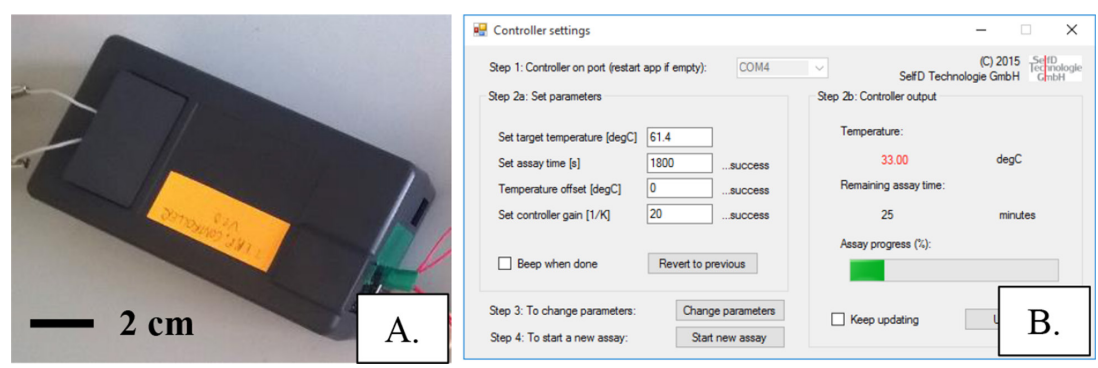

Figure 1: Mini-thermostat (a) and user interface (b). 

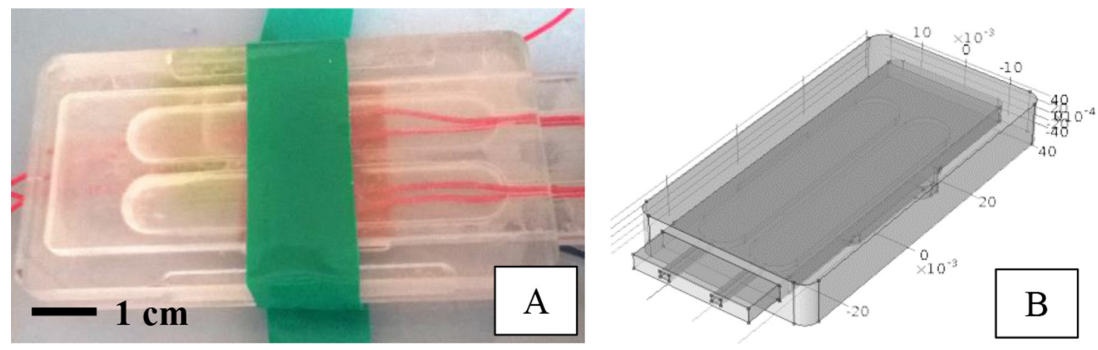

Figure 2: Test structure (a) and model geometry (b).

to form a heating element, which is sandwiched between layers of electrical insulation. The sample used in the experimental setup was an etched foil heater from Minco Products, Inc. (P/N HR5303R70.2LI2A), in which the etched resistive film was encased in polyimide and silicon rubber sheets.

According to manufacturer specifications [9], the resistance of this heating element was $70.2 \Omega$. Temperature output of the heating element was controlled by the mini-thermostat described in Section 3.1. The thermostat regulated current input from a DC power supply (Agilent E3631A). A milled poly-methylmethacrylate (PMMA) test structure was used to emulate a Point-of-Care device with a disposable microfluidic chip $(25 \mathrm{~mm} \times 75 \mathrm{~mm})$ that contained microreactor cavities. Both channels contained NTC 10k thermistors, one wired to the mini-thermostat, and the other to a Multimeter (Agilent 34410A) for independent temperature recording (Fig. 2a). The power supply was set up, a temperatures logged using a MATLAB script.

\subsection{Setup \#3}

This experimental setup was similar in every regard to Setups \#1 and \#2 described in Section 3.2, except the power supply, which was a set of 2 pcs. of $9 \mathrm{~V}$ batteries (GP 1604A).

\section{RESULTS AND DISCUSSION}

\subsection{Electrical characterization of heating element}

Manufacturer specifications state the resistance of the heating element as $70.2 \Omega$, however, in a previous work we measured initial resistance to be $76.38 \pm 0.37 \Omega$ [3].

\subsection{Model evaluation}

\subsubsection{Setup \#1}

Nucleic acid amplification reactions are currently the gold standard in molecular diagnostics assays for the detection of certain living organisms, suchs pathogens. Isothermal amplification reactions require a single temperature or temperature range to be maintained for the specified assay time (15-60 minutes).

Model and controller performance were evaluated on temperature targets defined for various isothermal nucleic acid amplification assays in the literature (see Table 1, Craw [10]). The maximum temperature required by these assays, $65^{\circ} \mathrm{C}$, was well below the deflection 
Table 1: Target temperatures for isothermal nucleic acid amplification assays.

\begin{tabular}{lll}
\hline Method & Full name & Temperature target $\left({ }^{\circ} \mathrm{C}\right)$ \\
\hline NASBA & Nucleic Acid Sequence Based Amplification & 41 \\
HDA & Helicase dependent amplification & 64 \\
LAMP & Loop mediated isothermal amplification & $60-65$ \\
NEAR & Nicking Enzyme Amplification Reaction & $55-59$ \\
RCA & Rolling Circle Amplification & $30-65$ \\
RPA & Recombinase Polymerase Amplification & $37-42$ \\
SPIA & Single primer isothermal amplification & $45-50$ \\
RAM & Ramification amplification method & 35 \\
\hline
\end{tabular}

temperature of the plastic we used in the experimental setups. Room temperature for the model was defined as $19^{\circ} \mathrm{C}-22^{\circ} \mathrm{C}$, and the thermistor baseline for temperature recording as $26^{\circ} \mathrm{C}$ (see Section 3.2 for more details).

The model was validated using the Joule Heating interface of COMSOL® Multiphysics Version 5.2 using the model geometry shown in Fig. 1b. Material properties were derived from the built-in libraries of the software package. The mini-thermostat received a $15 \mathrm{~V}$ input with 1 A current limit from the power supply, and the controller gain $K_{p}$ was set to 15 [1/K]. Set temperatures were according to Table 1 and Fig. 3, taking lower and upper ends of temperature ranges as well as single target temperatures where appropriate. To evaluate model performance, the same parameters were fed into the model and a comparison made.

The model was validated using a PC with a Core i5-4570 CPU and 16 GB RAM. Solution time varied with set temperatures, showing a nearly linear increase in solution time with tem-

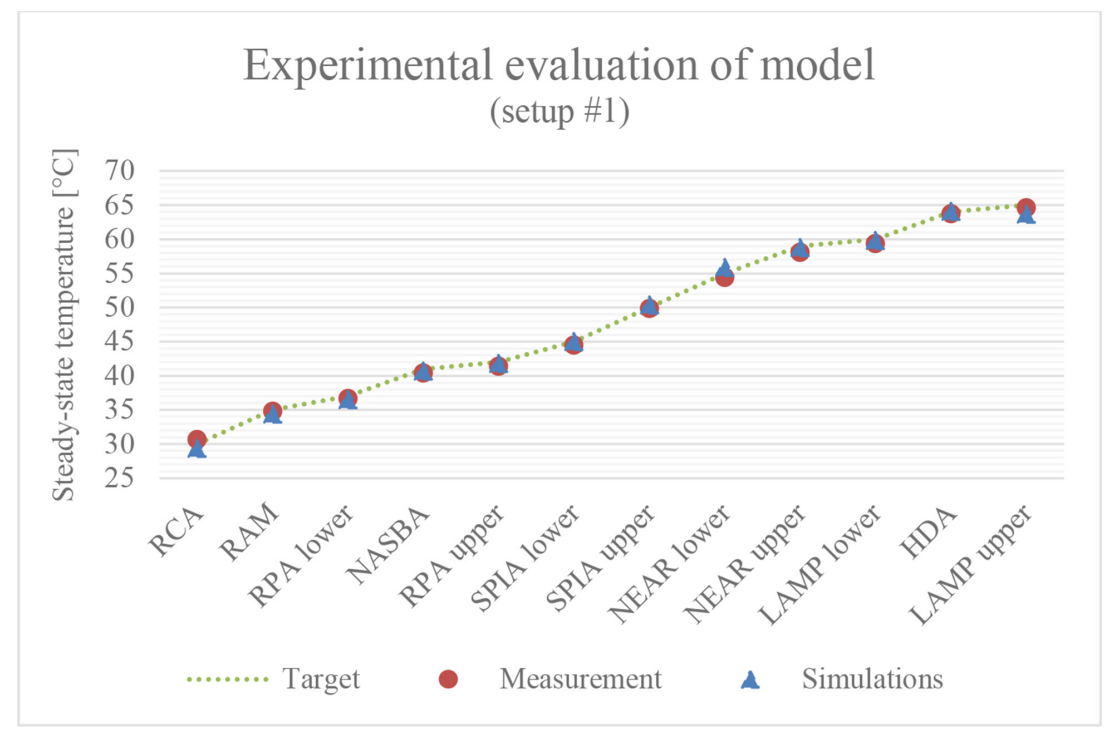

Figure 3: Experimental evaluation of setup \#1. 
perature increase. For a set temperature of $30^{\circ} \mathrm{C}$, the model was validated in 14 minutes 42 seconds ( \pm 1 minute 43 seconds), whereas for $65^{\circ} \mathrm{C}$, solution time was 55 minutes 25 seconds ( \pm 31 minutes 8 seconds). This difference was caused by an increase in complexity: at lower set temperatures, steady-state was reached in a shorter time, after which the length of time steps could be longer and, therefore, the model took a shorter time to solve, as there was no significant change. The variance of solution times is also due to a varying load on the computer from other tasks running in parallel.

Temperatures inside the experimental setup were recorded as described in Section 3.2, with an independent thermistor in a cavity adjacent to the sensor connected to the thermostat. Appropriate temperature offsets were applied to compensate for errors resulting from the different physical positions of the two sensors. Each recording was conducted for $30 \mathrm{~min}$ utes, during which steady-state was possible to achieve $\left( \pm 1{ }^{\circ} \mathrm{C}\right.$ around the set temperature). On average, steady-state temperatures recorded in the microreactor cavity of the experimental setup were within $0.53^{\circ} \mathrm{C} \pm 0.22^{\circ} \mathrm{C}$ from the designated set temperatures derived from Table 1. The finite element model estimated recorded temperature outputs with an error of $0.64^{\circ} \mathrm{C} \pm 0.39^{\circ} \mathrm{C}$. Comparison of results is shown in Fig. 3 .

\subsubsection{Setup \#2}

For this evaluation, the LAMP (loop-mediated isothermal amplification) reaction, an isothermal nucleic acid amplification method was taken into account. This is a highly sensitive and specific amplification method particularly suitable for use in molecular diagnostics, developed by Eiken Chemical Co. $[10,11]$, which requires a $60^{\circ} \mathrm{C}-65^{\circ} \mathrm{C}$ temperature range to be maintained for 15-60 minutes for a successful amplification of DNA samples.

The model was validated using the Joule Heating interface of COMSOL® Multiphysics Version 5.2 using the same model geometry and material properties as setup \#1. The mini-thermostat received an $18 \mathrm{~V}$ input with $1 \mathrm{~A}$ current limit from the power supply, and the controller gain $K_{p}$ was set to $20[1 / \mathrm{K}]$. Set temperature was $63.8^{\circ} \mathrm{C}$. To evaluate model performance, experimental parameters were fed into the model and a comparison made.

The model was solved using a PC with a Core i5-4570 CPU and 16 GB RAM with an average solution time of 1 hour 43 minutes ( \pm 10 minutes 45 seconds).

Temperature recordings in this experimental setup indicated a more pronounced controller error $\left(1.54^{\circ} \mathrm{C} \pm 0.09^{\circ} \mathrm{C}\right)$ in steady-state than was observed in setup \#1 (Section 4.2.1). This was due to the larger controller gain and higher input voltage, and was repeatedly observed during measurements. In a side-by-side comparison of model and experimental results, the mean absolute error was $2.02^{\circ} \mathrm{C}\left( \pm 2.7^{\circ} \mathrm{C}\right)$ along the whole length of the recorded time period, whereas in steady-state, this error decreased to $0.52^{\circ} \mathrm{C}\left( \pm 0.19^{\circ} \mathrm{C}\right)$. Comparison of results is shown in Fig. 4.

\subsubsection{Setup \#3}

Battery-based operation was evaluated with conditions matching those of setup \#1 (Section 0 ), for temperature targets required by certain isothermal nucleic acid amplification reactions. A smaller set of temperature targets were chosen from Table 1 for this evaluation. Two pcs. of (fresh) $9 \mathrm{~V}$ batteries were used as power source and, therefore, input voltage was set to $18 \mathrm{~V}$ in the model.

Hardware and software used for validating the model was the same as for setup \#1, which led to similar solution times (see Section 0). 


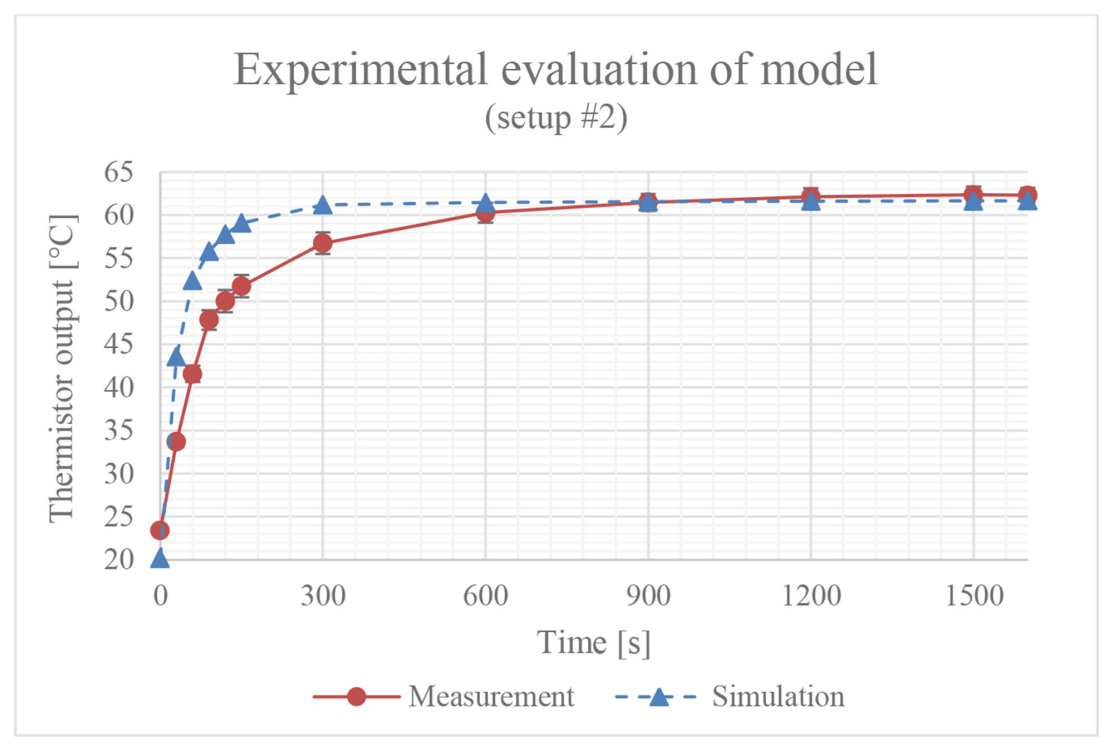

Figure 4: Experimental evaluation of Setup \#2.

Temperatures in the experimental setup were recorded as in setup \#1. Experimental results indicated that the controller was able to maintain target temperatures within the microreactor cavity with an average error of $0.05^{\circ} \mathrm{C} \pm 0.09^{\circ} \mathrm{C}$ after steady-state was reached. The decrease in error compared to setup \#1 (Section 4.2.1) was due to the smaller number of data points. The finite element model estimated recorded temperature outputs with an error of $0.69^{\circ} \mathrm{C}$ $\pm 0.34^{\circ} \mathrm{C}$. Although initially the output was $18 \mathrm{~V}$, battery voltage output decreased over the course of several experiments, which was not accounted for in the model. A comparison of the results is shown in Fig. 5.

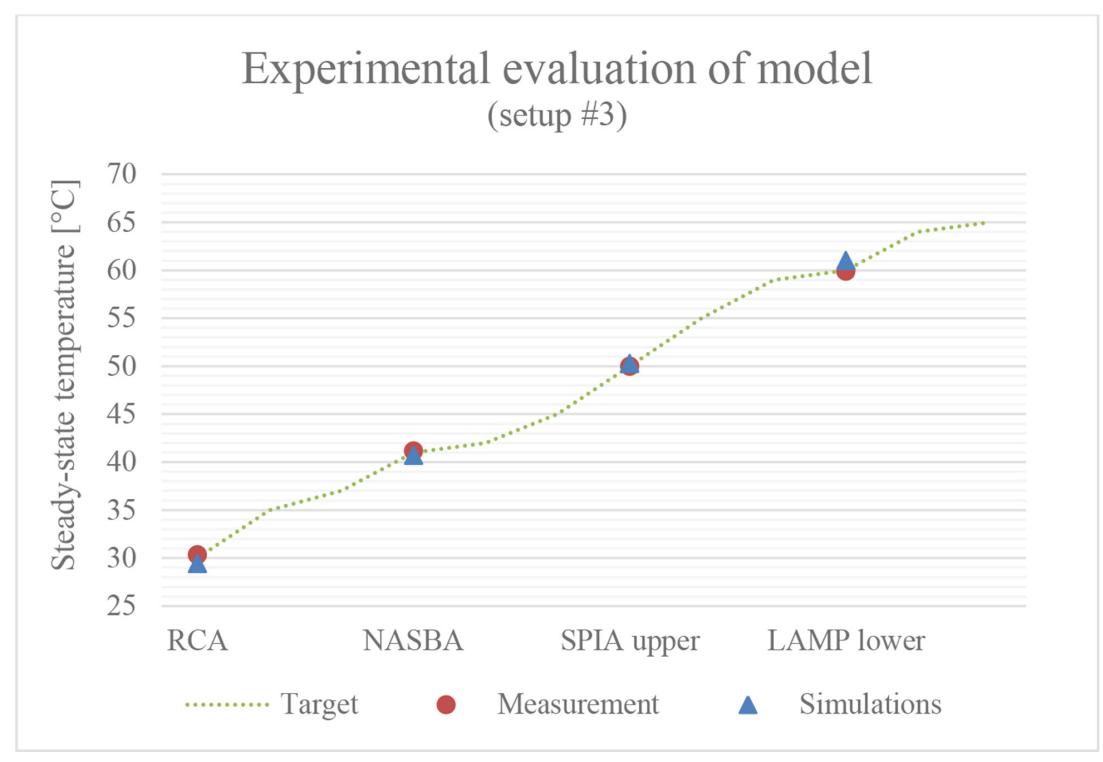

Figure 5: Experimental evaluation of setup \#3. 


\section{CONCLUSIONS}

A finite element model was proposed for the modelling of actively controlled resistive heating elements for applications in microheating solutions for disposable molecular diagnostics devices to aid in iterative optimization and in silico validation of device designs. The model was presented and evaluated for experimental setups imitating a disposable molecular diagnostics platform with temperature control and a replaceable cartridge (chip) with a microfluidic reactor cavity, matching the typical dimensions of such devices. For temperature control purposes, the heat output of a commercially available etched foil resistive heating element was controlled using a mini-thermostat developed for open-source Arduino-compatible hardware.

Target temperatures were chosen to match several molecular diagnostics assays (isothermal nucleic acid amplification assays). The proposed finite element model was capable of approximating temperature outputs recorded in the experimental setups with an error of $0.64^{\circ} \mathrm{C} \pm 0.39^{\circ} \mathrm{C}$ over a range of different target temperatures between $30^{\circ} \mathrm{C}$ and $65^{\circ} \mathrm{C}$ when operated from a DC power supply. In a side-by-side comparison, approximation error was $0.52^{\circ} \mathrm{C} \pm 0.19^{\circ} \mathrm{C}$. During battery operation, the model was capable of estimating experimentally recorded temperatures with an average error of $0.69^{\circ} \mathrm{C} \pm 0.34^{\circ} \mathrm{C}$.

Furthermore, the proposed mini-thermostat device was capable of controlling temperature outputs with $0.53^{\circ} \mathrm{C}$ precision around 12 distinct set temperatures from $30^{\circ} \mathrm{C}$ to $65^{\circ} \mathrm{C}$ during 30 minutes of operation, when operated both from a DC power supply and batteries.

To summarize, the proposed model, despite being in early stages of development, was capable of approximating time-dependent heat transfer in a disposable molecular diagnostics device with more than $1{ }^{\circ} \mathrm{C}$ precision. Additionally, a mini-thermostat based on open-source hardware was proposed and demonstrated to be able to support isothermal nucleic acid amplification assays in disposable Lab-on-a-Chip devices.

\section{ACKNOWLEDGEMENTS}

The authors wish to thank the support of the Sächsische AufbauBank provided under project SAB\#100204668, the Estonian Research Council provided under research project IUT1911 and the Horizon 2020 ERA-chair initiative VFP5051 'Cognitive Electronics - COEL'. Additionally, the authors would like to express their gratitude to Jüri Oleitšuk for the manufacturing of test structures.

\section{REFERENCES}

[1] Moschou, D., Vourdas, N., Kokkoris, G., Papadakis, G., Parthenios, J., Chatzandroulis, S. \& Tserepi, A., All-plastic, low-power, disposable, continuous-flow PCR chip with integrated microheaters for rapid DNA amplification. Sensors and Actuators B: Chemical, 199, pp. 470-478, 2014. http://dx.doi.org/10.1016/j.snb.2014.04.007

[2] Wang, Y., Song, H. \& Pant, K., A reduced-order model for whole-chip thermal analysis of microfluidic lab-on-a-chip systems. Microfluidics and Nanofluidics, 16(1-2), pp. 369-380, 2014. http://dx.doi.org/10.1007/s10404-013-1210-0

[3] Pardy, T., Tulp, I. \& Rang, T., Finite element modelling of the resistive heating of disposable molecular diagnostics devices. Computational Methods and Experimental Measurements XVII, 59, pp. 381-391, 2015.

http://dx.doi.org/10.2495/cmem150341 
[4] Srivastava, M., Srivastava, M.C. \& Bhatnagar, S., Control Systems, Tata McGraw-Hill: New Delhi, Singapore, 2009.

[5] Meier, A.V., Electric Power Systems: A Conceptual Introduction, IEEE Press, WileyInterscience, Hoboken, N.J, 2006.

[6] Griffiths, D.J., Introduction to electrodynamics. Prentice Hall, 93, p. 95, 1999.

[7] Kandlikar, S.G., Garimella, S., Li, D., Colin, S. \& King, M.R., Heat Transfer and Fluid Flow in Minichannels and Microchannels, Elsevier, 2006.

[8] Rood, P., A visual method of showing the high temperature coefficient of resistance of metals as compared with alloys. Journal of the Optical Society of America, 16(5), pp. 357-359, 1928.

http://dx.doi.org/10.1364/JOSA.16.000357

[9] Minco Products, Inc., Flexible heaters design guide HDG01121806(A), Minco Products Inc., 2007.

[10] Craw, P. \& Balachandran, W., Isothermal nucleic acid amplification technologies for point-of-care diagnostics: a critical review. Lab on a Chip, 12(14), pp. 2469-2486, 2012.

http://dx.doi.org/10.1364/JOSA.16.000357

[11] Nagamine, K., Hase, T. \& Notomi, T., Accelerated reaction by loop-mediated isothermal amplification using loop primers. Molecular and Cellular Probes, 16(3), pp. 223 229, 2002.

http://dx.doi.org/10.1006/mcpr.2002.0415 